\title{
Page Kidney: A Case of Acute or Chronic Renal Failure \& Refractory HTN Presenting after Renal Biopsy
}

\author{
Stephen Meyer, Aswin Nukala, Nikita Maniar*, Waldo Herrera \\ Department of Internal Medicine, Mt. Sinai Hospital, Chicago, USA \\ Email: "nikita.maniar@sinai.org
}

Received 19 February 2015; accepted 6 June 2015; published 9 June 2015

Copyright (C) 2015 by authors and Scientific Research Publishing Inc.

This work is licensed under the Creative Commons Attribution International License (CC BY). http://creativecommons.org/licenses/by/4.0/

(c) (7) Open Access

\begin{abstract}
Page Kidney is a relatively rare cause of Acute Renal Failure (ARF) presenting as accelerated and uncontrolled hypertension secondary direct compression of the renal parenchyma by an extrinsic source. This case report describes a 44-year-old male with advanced acute renal failure requiring hemodialysis, hypertension, and initial suspicion for thrombotic thrombocytopenic purpura who developed a case of Page Kidney following retroperitoneal hematoma following a renal biopsy. The patient was medically managed with intravenous nifedipine until blood pressure stabilized after improvement of the hematoma. Usually hematomas are self-resolving, however rarely they can result in the Page phenomenon-extrinsic compression of the affected kidney by the hematoma resulting in a picture that is similar to acute renal failure (ARF). This case highlights the importance of early medical management of blood pressure control after renal compression has been identified.
\end{abstract}

Keywords

Page Kidney, Acute Renal Failure, Refractory Hypertension

\section{Introduction}

Page kidney is a relatively rare cause of ARF and accelerated hypertension secondary to compression of the renal parenchyma often by intra-capsular hematoma leading to activation of the RAAS. Page kidney was first discovered in a canine experiment in which Irvin Page wrapped kidneys in cellophane and noted the induction of hypertension by peri-nephritis and compression of the renal parenchyma [1]. The pathophysiology of Page Kidney

\footnotetext{
"Corresponding author.
}

How to cite this paper: Meyer, S., Nukala, A., Maniar, N. and Herrera, W. (2015) Page Kidney: A Case of Acute or Chronic Renal Failure \& Refractory HTN Presenting after Renal Biopsy. Open Journal of Nephrology, 5, 40-43. 
involves external compression of the renal parenchyma by a mass often resulting from trauma. This leads to persistent activation of the renin-aldosterone system resulting in accelerated hypertension that tends to be refractory [2]. This injury has been reported in the literature as presenting after trauma from Motor Vehicle Accidents, sports injuries, or after renal biopsies. As is seen in cases of renal artery stenosis, when perfusion to the kidney is diminished, the renin-angiotensin pathway is activated resulting in an elevation in blood pressure to increase renal perfusion [3]. In Page Kidney, this pathway is persistently activated resulting in acute onset accelerated hypertension.

\section{Case Report}

A 44-year-old African American male with past medical history of migraine headaches, ARF requiring hemodialysis, hypertension, and recent suspicion thrombotic thrombocytopenic purpura (TTP) not responding to weekly plasmapheresis presented to our institution as a transfer from an LTAC facility due to down-trending platelet counts and further workup of recently suspected TTP. Five months prior to admission, the patient had presented to an outside hospital with complaints of headache. At that time he was found to have ARF and uncontrolled hypertension which required ICU admission and hemodialysis. The patient had a prolonged hospitalization during which there was a suspicion of TTP causing his acute medical conditions and was started on plasmapheresis and corticosteroid therapy, stabilized and transferred to an LTAC. He was further started on hemodialysis three times per week for his ARF, and plasmapheresis twice weekly for suspicion of TTP while at the LTAC. He presented to our institution because his CBC showed down-trending platelets without any active bleeding and because his TTP workup was incomplete. Upon admission to our institution, the patient was hemodynamically stable, blood pressure was 140's systolic. Labs were significant for a BUN of 68 and Creatinine of 8.8. Other electrolytes were within normal limits. $t$ was agreed that further workup would be needed to determine the cause of his ARF.

Nephrology and hematology were consulted on the case and a renal biopsy was agreed upon as the next step in management after labs including LDH, ANCA, Anti-GBM, Complements C3/C4, ANA, ADAMTS-13, hepatitis panel and HIV were negative. On admission day 2, the patient underwent ultrasound guided biopsy of the left kidney, and on admission day 3 , he began having severe left lower quadrant abdominal and suprapubic pain, with an appreciable drop in hemoglobin from 8.9 to 5.4 within a 24-hour period. His blood pressure acutely elevated with from167/91 to 213/116, HR 104.CT abdomen/pelvis revealed a large complex left retroperitoneal hematoma extending along the left para-colic gutter and into the pelvis (Figure 1). An ultrasound of the kidneys again revealed the mass without any focal changes in the kidneys themselves.

Surgical consultants recommended conservative management and the patient was controlled with PRN transfusions to maintain adequate hemoglobin and the patient was subsequently admitted to the MICU service on day 4 and started on nicardipine drip for management of accelerated hypertension refractory to oral antihypertensive medical management until further interval resolution of the hematoma occurred and hypertensive management was able to be de-escalated.

Renal biopsy revealed severe interstitial fibrosis and tubular atrophy involving the entire cortex, severe arterial sclerosis and arteriolar hyalinosis consistent with hypertension and NSAID use which the patient admitted he had been taking for his migraine headaches. The patient's medication was adjusted and his blood pressure was refractory requiring high doses of clonidine, nifedipine, coreg, hydralazine, minoxidil, and furosemide. The patient came to our institution with a known history of hypertension, however his acute refractory surge in blood pressure after development of a renal hematoma, enabled a diagnosis of Page Kidney as a complication of the renal biopsy and then was confirmed by CT imaging. The expected outcome for this patient was favorable and outpatient follow-up was established which has further allowed for further de-escalation of antihypertensive medication and now he is controlled on only two oral antihypertensives.

\section{Discussion}

Renal hematoma can be managed either surgically or medically. Acute onset of flank/abdominal pain, tenderness, as well as an acute drop in hemoglobin and hematocrit after a potential injury should all raise concern for retroperitoneal bleed [4]. Many cases of Page Kidney can be successfully managed by controlling the associated hypertensive disorder [5]. A CT scan without contrast should be ordered immediately followed by prompt surgical evaluation. In the Page phenomenon the rapid acute rise in blood pressure can be very dangerous-resulting 


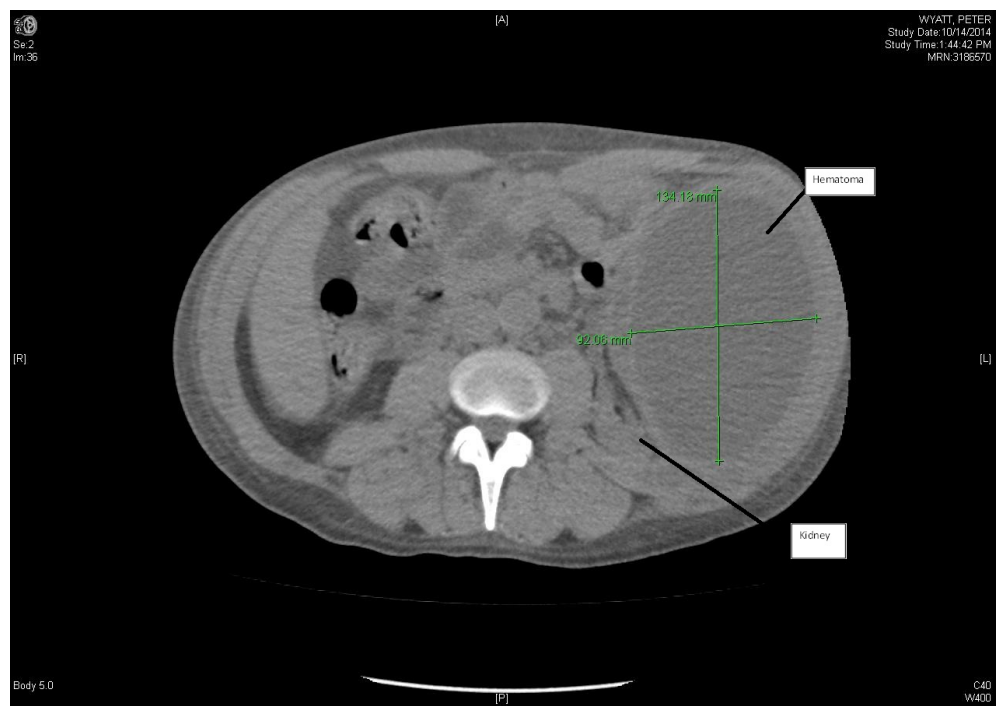

Figure 1. Retroperitoneal hematoma post left renal biopsy.

in stroke, MI, or worsening of the bleed itself [6]. In this case, we advocate the importance of rapid blood pressure control with intravenous anti-hypertensive medication to prevent the size of the hematoma from progressing, thereby worsening the Page phenomenon [7]. Oral medications can be unpredictable in this scenario and often times may not be tolerated by the patient due to the severe abdominal pain [8]. The calcium channel blocker nicardipine, was successful in this case and may be considered a good choice as it can be administered intravenously in an ICU setting with close monitoring, and can be titrated quickly to maintain a systolic blood pressure less than 140. After 24 - 48 hours of strict control, the patient should be reassessed clinically to determine resolution of symptoms. A repeat CT scan should then be ordered to reassess the size of the bleed. If stable, the patient can then be safely transitioned to oral medications. As the hematoma resolves, the hypertension and ARF will also resolve [9].

\section{Conclusion}

In conclusion, we have reported a case of Page Kidney that has been successfully treated with medical management. There have been studies showing the use of anti-hypertensive medications to manage subcapsular hematomas, in our case of an extracapsular bleed the same medical management was used to resolve the hematoma [10]. The patient's clinical course was favorable with complete resolution of symptoms and stabilization on oral medications. The patient currently comes to the clinic for follow-up of his hypertension and kidney disease.

\section{References}

[1] Smyth, A., Collins, C., Thorsteinsdottir, B., Madsen, B., Oliveira, G., Kane, G. and Garovic, V. (2012) Page Kidney: Etiology, Renal Function Outcomes and Risk for Future. The Journal of Clinical Hypertension, 14, 216-221. http://dx.doi.org/10.1111/j.1751-7176.2012.00601.x

[2] Kim, M., Parks, K. and Miller, E. (2013) Page Kidney: Hypertension Secondary to Extra-Renal Compression. Journal of Diagnostic Medical Sonography, 29, 26-29. http://dx.doi.org/10.1177/8756479312457593

[3] Dopson, S., Jayakumar, S. and Velez, J. (2009) Page Kidney as a Rare Cause of Hypertension: Case Report and Review of the Literature. American Journal of Kidney Diseases, 25, 334-339. http://dx.doi.org/10.1053/j.ajkd.2008.11.014

[4] Mathew, A., Brahmbhatt, B., Rajesh, R., Kurian, G. and Unni, V.N. (2009) Page Kidney. Indian Journal of Nephrology, 19, 170-171. http://dx.doi.org/10.4103/0971-4065.59342

[5] Brotfain, E., Koyfman, L., Frenkel, A., Smolikov, A., Zlotnik, A. and Klein, M. (2013) Traumatic Page Kidney Induced Hypertension in Critical Care: Immediately Resolved or Long-Term Resistant Problem. Case Reports in Critical Care, 2013, Article ID: 201424. http://dx.doi.org/10.1155/2013/201424

[6] Chung, J., Caumartin, Y., Warren, J. and Luke, P. (2008) Acute Page Kidney Following Renal Allograft Biopsy: A 
Complication Requiring Early Recognition and Treatment. American Journal of Transplantation, 8, 1323-1328. http://dx.doi.org/10.1111/j.1600-6143.2008.02215.x

[7] Nakano, S., Kigoshi, T., Uchita, K., Morimoto, S., Tsugawa, R. and Matsunou, H. (2008) Hypertension and Unilateral Renal Ischemia (Page Kidney) Due to Compression of a Retroperitoneal Paraganglioma. American Journal of Nephrology, 16, 91-94. http://dx.doi.org/10.1159/000168976

[8] Myrianthefs, P., Aravosita, P., Tokta, R., Louizou, L., Boutzouka, E. and Baltopoulos, G. (2007) Resolution of Page Kidney-Related Hypertension with Medical Therapy: A Case Report. Heart \& Lung: The Journal of Acute and Critical Care, 36, 377-379. http://dx.doi.org/10.1016/j.hrtlng.2006.10.009

[9] Kapil, S., Ashish, S., Viswaroop, S., Arul, M. and Gopalakrishnan, G. (2011) Page Kidney—Rare but Correctable Cause of Hypertension. UroToday International Journal, 4, art54. http://dx.doi.org/10.3834/uij.1944-5784.2011.10.10

[10] Patel, T.V. and Goes, N. (2007) Page Kidney. Kidney International, 72, 1562.

http://dx.doi.org/10.1038/sj.ki.5002580 